\title{
ALDONA Sopata
}

\section{Funktionale Kategorien im Erwerb des Deutschen und des Polnischen als Erstsprache(n) ${ }^{1}$}

\begin{abstract}
Uczenie się języka ojczystego jest fascynującym procesem, który jest badany z wykorzystaniem wielu teorii lingwistycznych. Opis mentalnej gramatyki człowieka, jaki dostarcza nam teoria generatywna, pozwala na bardzo szczegółowe badanie tego fenomenu. Niniejszy artykuł przedstawia rozwój kategorii funkcjonalnych podczas akwizycji języka niemieckiego i polskiego jako języka ojczystego. Porównanie tego procesu w obu językach wspiera hipotezę przedstawiająca proces rozwoju języka pierwszego jako stopniowy rozwój struktury językowej w umyśle dziecka.

Das Erlernen der Muttersprache ist ein faszinierender Prozess, der auf der Grundlage verschiedener linguistischer Theorien untersucht wird. Die Beschreibung der mentalen Grammatik des Menschen, die durch die Generative Theorie geliefert wird, ermöglicht eine sehr detaillierte Untersuchung des Phänomens. Der vorliegende Artikel stellt die Entwicklung der funktionalen Kategorien im Erwerb des Deutschen und des Polnischen als Muttersprache(n) dar. Ein Vergleich des Prozesses in beiden Sprachen unterstützt die Hypothese, die den Erstspracherwerb als eine graduelle Entwicklung der Sprachstruktur im kindlichen Gehirn erklärt.
\end{abstract}

Learning of the mother tongue is a fascinating process, which is investigated on the basis of various linguistic theories. The description of the human mental grammar delivered by Generative Theory allows for a very detailed investigation into the phenomenon. The present article describes the development of functional categories in the acquisition of German and Polish as first language. A comparison of the process in both languages supports the hypothesis explaining the first language acquisition in terms of a gradual development of the language structure in a child's mind.

\footnotetext{
Der Artikel entstand in einem von der Alexander von Humboldt-Stiftung geförderten Projekt. Für Anregungen und Kommentare zum Beitrag möchte ich mich bei Prof. Jürgen M. Meisel und Prof. Alicja Nagórko bedanken.
} 
Aldona Sopata

\section{Einführung}

Der Prozess des Erwerbs der Muttersprache von Kindern ist ein Phänomen, das viele Forscher staunen lässt. Ungefähr an seinem ersten Geburtstag formuliert ein Kind seine ersten Worte. Im zweiten Lebensjahr produziert es Zweiwort- und dann Mehrwort-Kombinationen, und im dritten entwickelt es das Hauptgerüst seiner Muttersprache, auch wenn dieses noch nicht perfekt ist. Wie Untersuchungen belegen (u.a. OTSU 1981), sind schon Kindern im Alter von 3 Jahren Phrasen als syntaktische Einheiten verfügbar. ${ }^{2}$ Im Gegensatz zum Erlernen von Fremdsprachen brauchen Kinder keinen Sprachunterricht, um ihre Muttersprache zu erwerben. Die Eltern sprechen mit ihren Kindern nicht über die Sprachregeln und erklären ihnen nicht, wie man beispielsweise einen Satz aufbauen soll. Die Sprache der Kinder entwickelt sich spontan, aufgrund des sogenannten sprachlichen Inputs, bzw. der primären Sprachdaten, d.h. auf Basis dessen, was Kinder hören. Sie werden auch selten von den Eltern korrigiert, und selbst wenn eine solche Korrektur erfolgt, reagieren sie kaum darauf (vgl. u.a. GlEITMAn / WANNER 1982). Wenn ein Kind von den Eltern korrigiert wird, dann betrifft dies fast immer den Inhalt der kindlichen Äußerungen und nicht deren formale Korrektheit (CAZDEN 1972; PINKER 1994). Elterliche Korrekturen könnten dem Kind Auskunft darüber geben, was in der zu erwerbenden Sprache nicht möglich ist. Da Korrekturen jedoch offensichtlich nur selten stattfinden und den Erwerb auch nicht nachweisbar beeinflussen, gehen die Spracherwerbsforscher davon aus, dass die Muttersprache nur aufgrund der sogenannten positiven Evidenz erworben wird. ${ }^{3}$

Diese Beobachtungen werden unter dem Begriff, Unterdeterminiertheit des grammatischen Wissens ' der Kinder durch die ihnen verfügbare Evidenz erfasst. Die quantitative Unterdeterminiertheit besagt, dass die sprachliche Erfahrung des Kindes stets nur einen relativ kleinen Ausschnitt der in einer Sprache möglichen Sätze und Strukturen umfasst. Das von den Kindern erworbene Wissen ist auch qualitativ unterdeterminiert, da es offensichtlich im Wesentlichen aus Regeln und Prinzipien besteht, während die sprachliche Erfahrung aus konkreten Äußerungen besteht, die lediglich als Exemplifizierung des zu

2 Heute wird diese Entwicklungsstufe noch früher angesetzt. Siehe dazu die nächsten Kapitel.

3 Obwohl Kinder keine Information darüber bekommen, welche Sätze in ihrer Muttersprache unzulässig sind, erwerben sie offensichtlich dennoch dieses Wissen, denn alle erwachsenen Sprecher einer Sprache sind imstande zu sagen, ob ein Satz grammatisch richtig oder falsch ist. 
erwerbenden Wissens gelten können. Da ein Kind an den ihm verfügbaren Daten erkennen kann, ob eine Struktur grammatisch ist (positive Evidenz), es jedoch nicht eindeutig erkennen kann, ob eine Struktur ungrammatisch ist (wenn eine Struktur in den gehörten Äußerungen nicht vorkommt, kann es ja bedeuten, dass sie einfach selten ist), spricht man von Unterdeterminiertheit durch das Fehlen negativer Evidenz. Dieses logische Problem des Spracherwerbs kann als Spezialfall von Platons Problem gelten: „In dem Nichtwissenden also sind von dem, was er nicht weiß, dennoch richtige Vorstellungen." (PLATON 1991:59) In der Spracherwerbsforschung besteht das Problem in der Frage, wie sprachliches Wissen und die Fähigkeit zu seiner Anwendung angesichts unterdeterminierter empirischer Evidenz von den Kindern erworben werden.

\section{Grundlagen der generativen Linguistik und der generativen Spracherwerbsforschung}

Auf die Frage, woher das sprachliche Wissen der Kinder kommt, geben unterschiedliche Ansätze innerhalb der Spracherwerbsforschung verschiedene Antworten. Die generative Spracherwerbsforschung löst das logische Problem des Erstspracherwerbs durch einen Verweis auf ein genetisch determiniertes Sprachprogramm. Der Erstspracherwerb ist als Wachstumsprozess zu verstehen, der zwar durch genetische Voraussetzungen determiniert ist, der aber zugleich von externen Faktoren abhängt, so wie auch andere biologische Entwicklungsprozesse in Abhängigkeit von solchen Faktoren (z.B. Ernährung) heranreifen (GREWENDORF 1992:13). Die Annahme der angeborenen Sprachfähigkeit erklärt, warum der Spracherwerb trotz aller Einschränkungen und Unterschiedlichkeiten der Lernbedingungen überhaupt möglich ist. Sie erklärt auch die Ähnlichkeiten im Verlauf und im Inhalt des Spracherwerbsprozesses - trotz der unterschiedlichen zu erlernenden Sprachen, trotz unterschiedlicher kognitiver Fähigkeiten der Kinder und trotz verschiedener Umstände, von denen der Erstspracherwerb begleitet wird.

Das genetisch festgelegte Sprachprogramm wird Universalgrammatik (UG) genannt (CHOMSKY 1981). Sie besteht aus universellen Prinzipien, die allen Sprachen gemeinsam sind, und sprachspezifischen Parametern. Die UG definiert das Ausmaß der möglichen strukturellen Variation und charakterisiert damit den Begriff einer möglichen menschlichen Sprache. Der Spracherwerb ist eine Interaktion zwischen den angeborenen Faktoren und der Umgebung. Die Architektur der UG wurde zuerst im Rahmen der Prinzipien- und Parametertheorie beschrieben (СHOMSKY 1981, 1986). Dann wurde sie in der neuesten 
Version der generativen Linguistik, dem Minimalistischen Programm, weiterentwickelt. Die universellen Prinzipien spiegeln die allen Sprachen gemeinsamen Eigenschaften wider. Als Beispiel kann das X-bar-Schema dienen. Als ein Teil der UG bestimmt es die allgemeine syntaktische Form einer Phrase. Eine Phrase ist hierarchisch aufgebaut und besteht aus drei Projektionsebenen (CHOMSKY 1981:48f.; HAEGEMAN 1991:103-106, 108-123). Nach diesem Prinzip sind alle Phrasen in allen natürlichen Sprachen so aufgebaut, dass sie stets einen lexikalischen Kopf (ein Schlüsselwort in der Phrase, dessen Merkmale die Eigenschaften der ganzen Phrase determinieren) enthalten müssen. Der Kopf bildet zusammen mit dem Komplement die nächsthöhere Kategorie $\mathrm{X}^{\prime}$, die sich wiederum mit einem Spezifikator zu X" verbindet. Ein Kind muss das X-bar-Schema nicht lernen, sondern es verfügt mit der UG über dieses (unbewusste) Wissen. Als Beispiel sei hier das X-bar-Schema für die folgende Determinantenphrase im Deutschen angeführt:

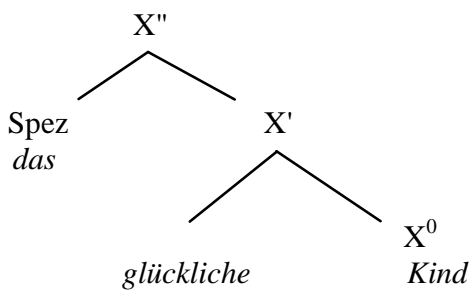

Die interne Struktur einer Phrase kann je nach Sprache variieren. Diese Unterschiede werden durch Parameter festgelegt. Der Kopfparameter legt beispielsweise fest, ob der Kopf einer Phrase rechts oder links stehen muss. Ein Beispiel für einen Parameter ist der sog. SVO/SOV- Parameter, der die Kopfstellung in der Verbalphrase festlegt und sich dadurch auf die interne Struktur des Satzes bezieht. Im Deutschen ist das dem Satz zugrundeliegende Wortstellungsmuster SOV (Subjekt-Objekt-Verb), was in den Nebensätzen zu sehen ist. ${ }^{4}$ Im Polnischen ist die Wortfolge SVO (Subjekt-Verb-Objekt) die kanonische Reihenfolge. Dieses Wissen muss ein Kind aufgrund des ihm verfügbaren sprachlichen Inputs erwerben. In der Prinzipien- und Parametertheorie geht man davon aus, dass in der UG zwischen lexikalischen und funktionalen Kategorien zu unterscheiden ist. Die lexikalischen Kategorien sind Nomen $(\mathrm{N})$, Verb (V), Adjektiv und Präposition. Abgesehen von den Präpositionen

\footnotetext{
4 Näher dazu im nächsten Abschnitt.
} 
bilden sie offene Klassen. ${ }^{5}$ Sie haben einen lexikalischen oder deskriptiven Inhalt. Funktionale Kategorien erfüllen dagegen eine grammatische Funktion (vgl. u. a. RADFORD 1997:45f., 66-68). Sie sind als Träger von grammatischen Merkmalen zu verstehen, wie beispielsweise die Merkmale Person, Numerus, Genus sowie Tempus- und Kasusmerkmale. Sie gehören geschlossenen Klassen von Elementen an, die nicht referentiell sind. Als Beispiel können hier die Kategorien der Determinierer und Teile des Flexionssystems genannt werden. Im verbalen Bereich werden in der Analyse von CHOMSKY (1986:160f.) eine Phrase des Komplementierers (CP) und eine Phrase der Flexion (IP) ${ }^{6}$ unterschieden. In der IP sind die Informationen über die Finitheit, also im Wesentlichen Tempus und Kongruenz, angesiedelt. Die Phrase der Flexion wird nach POLLOCK (1989) zudem in zwei Projektionen, Kongruenz (AGR) und Tempus (T), aufgeteilt. Ähnlich wie lexikalische Kategorien projizieren funktionale Kategorien auf Phrasen, die nach dem X-bar-Schema strukturiert sind. Funktionale Kategorien spielen eine besondere Rolle in der syntaktischen Strukturierung der Äußerung. Sie dominieren die lexikalischen Kategorien und bestimmen das Gerüst, in das inhaltliche Informationen eingesetzt werden, die aus lexikalischen Kategorien resultieren. Die nicht-referentiellen Artikel determinieren beispielsweise die Referenz von Nomina. Funktionale Kategorien können durch freie oder durch gebundene Morpheme realisiert werden. Zur ersten Gruppe gehören beispielsweise Determinierer, zur zweiten Kongruenzund Tempusmorpheme. Nach CHOMSKY (1989, 1995:232f.) sind nur funktionale Kategorien parametrisiert. Die Unterschiede zwischen den Sprachen sind auf unterschiedliche Eigenschaften der funktionalen Kategorien zurückzuführen. Der Erwerb der unterschiedlichen Eigenschaften von funktionalen Kategorien, die für eine gegebene Sprache charakteristisch sind, resultiert aus dem Erlernen unterschiedlicher grammatischer Phänomene ${ }^{7}$, die für die jeweilige Sprache spezifisch sind.

5 Die Präpositionen können in funktionale und lexikalische Kategorien eingeteilt werden.

6 Die Bezeichnung „Phrase der Flexion“ ist nicht ganz genau, s. MECNER (2005:130-134).

7 Für eine komplette Darstellung der Grundideen der generativen Sprachtheorie s. MeCNER (2005). 
Aldona Sopata

\section{Die Struktur des Deutschen und des Polnischen}

In dem Prinzipien- und Parametermodell werden eine zugrundeliegende und eine Oberflächenstruktur angenommen. Die in der VP generierten Elemente müssen in die Projektionen der funktionalen Kategorien, die die VP dominieren, bewegt werden, damit sie entsprechende Merkmale (Flexions- oder Kasusmerkmale) erhalten können. Aus diesen Bewegungen ergibt sich die Oberflächenstruktur. Im Minimalistischen Programm (CHOMSKY 1995:195f.) wird davon ausgegangen, dass die Elemente gleich mit den Merkmalen generiert werden. Sie müssen jedoch zur Überprüfung dieser Merkmale in funktionale Projektionen bewegt werden. Die Strukturbäume für unterschiedliche Sprachen sehen anders aus.

Das Deutsche wird als Verbzweit- / (V2)-Sprache auf Grund der Verbzweitstellung in Hauptsätzen analysiert. Wegen der Verbendstellung in Nebensätzen wird im Deutschen eine zugrundeliegende SOV (Subjekt-Objekt-Verb)-Stellung angenommen (s. z.B. GREWENDORF 2002:234-243). Nicht-finite Verben erscheinen am Ende sowohl im Haupt- als auch im Nebensatz. Es wird angenommen, dass die Verbalphrase (VP) und die Phrase der Flexion (IP) im Deutschen kopffinal sind. Während nicht-finite Verbalelemente in der VP bleiben, werden finite Verben in die IP bewegt, was in den Nebensätzen zu sehen ist (s. 2a). Die V2-Stellung der Hauptsätze kommt zustande, indem das finite Verb weiter in die Kopfposition (C) der Komplementiererphrase (CP) und das Subjekt in die Spezifziererposition dieser Phrase bewegt wird (s. 2b). Diese Bewegung wird dadurch ausgelöst, dass im Deutschen ein Merkmal für Finitheit [+F] in C steht (PlatzaCK / HolmberG 1989). Wenn die Kopfposition der CP lexikalisch durch einen Komplementierer gefüllt ist, bleibt das finite Verb in der rechtsköpfigen IP und bleibt am Satzende, was eben in den deutschen Nebensätzen passiert (s. 2a; vgl. u. a. PlatZACK / HOLMBERG 1989; GreWENDORF 1988:213-242, 2002:234-243). 
Funktionale Kategorien im Erwerb des Deutschen und des Polnischen

(1) Deutsche Struktur

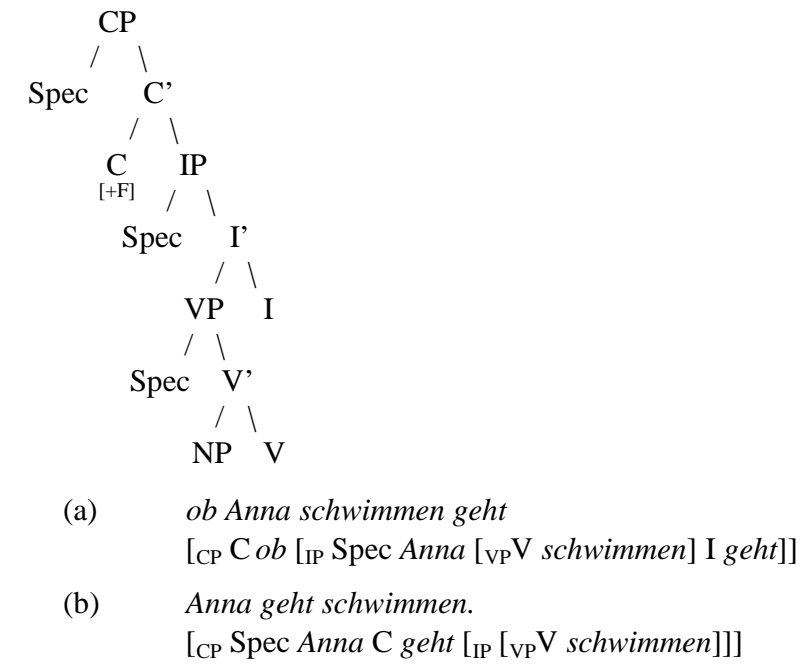

Das Polnische ist eine SVO-(Subjekt-Verb-Objekt)-Sprache. Die Phrasen VP und IP sind im Polnischen im Unterschied zum Deutschen linksköpfig. Wie im Deutschen wird das Verb im Polnischen auch in der Kopfposition der VP generiert. Da aber das Finitheitsmerkmal $[+\mathrm{F}]$ in der Kopfposition der IP lokalisiert ist, wird das Verb in die IP bewegt, um seine Flexionsmerkmale zu überprüfen, was im Polnischen mit einer anderen Wortfolge im Vergleich zum Deutschen zu erkennen ist (s. 4a, 4b ${ }^{8}$ ) (vgl. MECNER 1995, 1997, 2005:130138; PILARSKI 2002:92-100). ${ }^{9}$

8 Die unterschiedliche zugrundeliegende Struktur der Hauptsätze im Deutschen und im Polnischen ist auch an der Stellung der Negation zu erkennen. Im Deutschen ist sie postverbal und im Polnischen präverbal, was in der generativen Linguistik auf die unterschiedliche Position des Finitheitsmerkmals zurückgeführt wird (siehe dazu GREWENDORF 2002:40-46).

9 Im Rahmen des Artikels können nicht die alternativen Versuche diskutiert werden, die deutsche und polnische Struktur zu erklären. Die hier skizzierte Theorie ist der klassische Ansatz innerhalb der generativen Literatur. 
Aldona Sopata

(3) Polnische Struktur

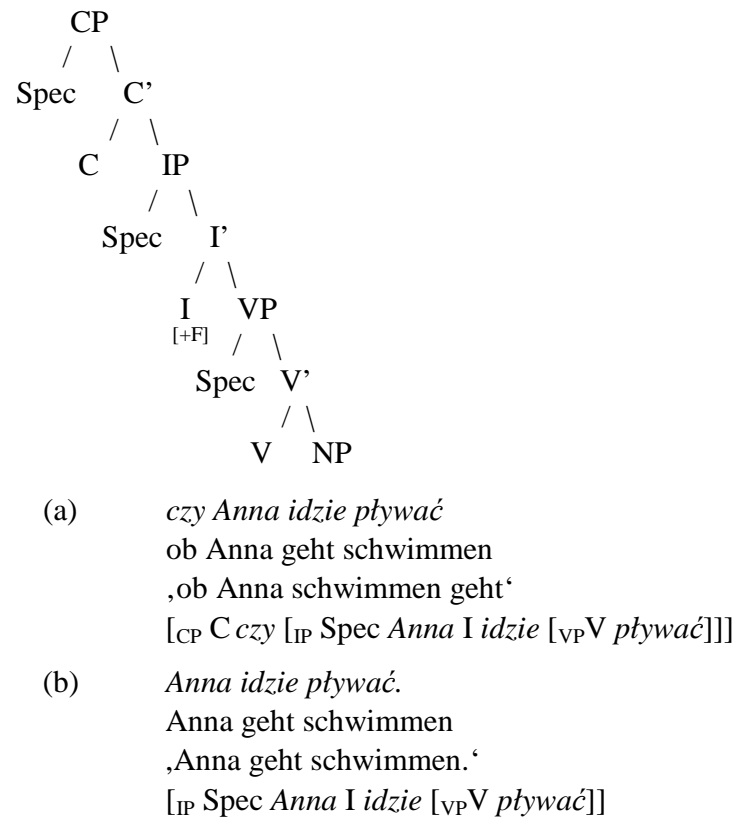

\section{Hypothesen zum Erstspracherwerb}

Die generative Theorie über die mentale Grammatik des Menschen ist Ausgangspunkt für mehrere Hypothesen zum Verlauf des Erstspracherwerbs. Grundsätzlich kann man in der Erstspracherwerbsforschung von zwei wissenschaftlichen Positionen sprechen. Einige Forscher (z.B. POEPPEL / WEXLER 1993) vertreten die Ansicht, dass Kinder- und Erwachsenenäußerungen strukturell gleich sind und alle funktionalen Kategorien dem Kind von Beginn an zur Verfügung stehen. Andere gehen von minimalen lexikalischen Projektionen in der frühen Kindersprache aus, wobei die vollständige Struktur der Erwachsenensprache durch Reifung (Reifungshypothese - BORER / WEXLER 1987) oder durch Strukturaufbau (Strukturaufbauhypothese - GUILFOYLE / NOONAN 1992) erreicht wird.

In der Kontinuitätshypothese geht PINKER (1984) davon aus, dass in der Kindersprache die gleichen Kategorien und die gleichen Relationen wie in der Erwachsenensprache verwendet werden. Der Spracherwerb verläuft nach der 
Kontinuitätshypothese in einer Reihe kontinuierlicher Stadien, und die den kindlichen Äußerungen zugrundeliegende Grammatik ist in jeder Entwicklungsstufe durch universelle Prinzipien determiniert (vgl. u.a. HYAMs 1987). Das Kind konstruiert nach dieser Hypothese eine UG-konforme Grammatik auf Basis der von ihm gehörten und verarbeiteten sprachlichen Äußerungen. Die Grammatiken in den einzelnen Sprachentwicklungsstufen können sich von der zielsprachigen Erwachsenengrammatik unterscheiden, sie stimmen jedoch immer mit den universellen Prinzipien einer möglichen natürlichen Sprache überein, so dass es nicht zu „wilden Grammatiken“ (GoODLUCK 1986) kommt. Das Kind gelangt von einer Erwerbsstufe in die nächste dank der Informationen, die es den gehörten und verarbeiteten sprachlichen Äußerungen entnimmt, wobei seine Grammatik entsprechend rekonstruiert wird.

POEPPEL / WEXLER (1993) sowie BORER / ROHRBACHER (2002) argumentieren, dass Kinder von Anfang an die volle Kompetenz besitzen. In der Kindergrammatik sind nach dieser Hypothese (Hypothese der vollen Kompetenz) alle funktionalen Kategorien von Beginn an vorhanden. Als Argument dafür deuten die Autoren eine Beobachtung, dass das von ihnen untersuchte Kind überwiegend finite Verbalformen verwendet und sie im Satz in die der Erwachsenengrammatik entsprechende Zweitstellung setzt. Die seltenen Fehler des Kindes werden als Performanzfehler betrachtet. POEPPEL / WEXLER (1993) plädieren auch dafür, dass die übergeordnete funktionale Kategorie $\mathrm{CP}$ gleich am Anfang der Sprachentwicklung vorhanden ist. Die Autoren sehen die Evidenz dafür in der Beobachtung, dass das Kind auch andere Elemente als Subjekte vor die finiten Verben stellt. Das kann in der Tat als Anzeichen für das Vorhandensein der übergeordneten funktionalen Kategorie gedeutet werden. Ungeklärt bleibt jedoch, wie die Abwesenheit von Komplementierern und Fragesätzen erklärt werden kann, wenn die Kategorie des Komplementierers gleich zu Beginn in der Sprachstruktur präsent sein sollte.

In der Reifungshypothese gehen FELIX (1984) sowie BORER / WEXLER (1987) davon aus, dass die Entwicklung universaler Prinzipien Teil eines genetischen Programms ist. Die Prinzipien reifen nach und nach heran. Die verschiedenen Sprachentwicklungsstufen des Kindes werden durch neurophysiologische Reifung erklärt. Frühe Äußerungen der Kinder können gegen universale Prinzipien verstoßen, weil die Prinzipien im kindlichen Gehirn noch „reifen“ müssen. Argumente für die Reifungshypothese sind Beobachtungen, die bei der Entwicklung anderer biologischer Eigenschaften von Menschen oder Tieren gemacht wurden. BORER / WEXLER (1987) weisen beispielsweise darauf hin, dass das Geschlecht des Menschen durch seine Gene festgelegt ist, sekundäre 
Aldona Sopata

Geschlechtsmerkmale jedoch erst im Verlauf der Pubertät erscheinen. Der Ansatz liefert eine Antwort auf eine schwierige Frage, nämlich die, wodurch das Setzen von bestimmten Parameterwerten zu einem bestimmen Zeitpunkt ausgelöst wird. Die Annahme, dass der Erstspracherwerb durch den neurophysiologischen Reifungsprozess determiniert wird, hat jedoch wegen des Mangels an einer unabhängigen Evidenz wenig Erklärungskraft (vgl. GUILFOYLE / NOONAN 1992). Durch die Hypothese der ,Reifung ' vermeidet man eher die Suche nach einer internen Logik der Spracherwerbsstadien. Nach dieser Hypothese sind in der Kindersprache auch ,wilde Grammatiken“"10 zu erwarten, weil einige universelle Prinzipien in den frühen Erwerbsstufen noch nicht herangereift seien. Der Erstspracherwerb verläuft aber sehr ähnlich bei allen Kindern (von pathologischen Fällen abgesehen), und kindliche Äußerungen scheinen immer UG-konform zu sein, was gegen die Annahme von ,wilden Grammatiken“ und damit auch gegen die Reifungshypothese spricht.

Als anderer Lösungsvorschlag für das Problem des Spracherwerbs kann die Strukturbildungshypothese von GUILFOYLE / NOONAN (1992) angesehen werden. Nach diesem Ansatz sind nur lexikalische Kategorien am Anfang des Spracherwerbs Teil der kindlichen Grammatik. Funktionale Kategorien werden dagegen erst sukzessive in die Grammatik implementiert (s. auch RADFORD 1990, 1995). Die Struktur der Kindersprache wächst nach und nach heran, wobei jede Stufe der Sprachentwicklung von den universellen Prinzipien bestimmt wird, so dass es nicht zu ,wilden Grammatiken“ kommt und die kindliche Grammatik nie gegen die UG verstößt. In bestimmten Entwicklungsstadien können jedoch einige Prinzipien noch nicht relevant sein, d.h. nicht anwendbar sein, weil die Voraussetzung für die Anwendung noch nicht erfüllt ist. Im Rahmen der Strukturaufbauhypothese wird also eine sukzessive Ergänzung der Grammatik durch immer weitere Struktureinheiten (Projektionen) vorgeschlagen. Unter anderem auf Basis der kindlichen Sprachdaten zum Erwerb der deutschen Wortstellung argumentieren GUILFOYLE / NOONAN (1992), dass die Kinder zunächst nur über lexikalische Kategorien wie Nomen oder Verb verfügen, funktionale Kategorien (z.B. Kategorie der Kongruenz oder der Flexion) und die mit ihnen verbundenen Projektionen dagegen erst in späteren Phasen der Sprachentwicklung erworben werden.

10 Als ,wilde Grammatiken“ werden Grammatiken bezeichnet, die mit den universellen Prinzipien einer möglichen natürlichen Sprache nicht übereinstimmen. Es sind also Grammatiken, die eine Äußerung wie Du wollen nicht Ball gut zulassen würden. Solche Äußerungen könnten eigentlich verstanden werden, werden jedoch von Kindern nie gemacht (vgl. GooDLUCK 1986). 
Die Annahme einer Reihenfolge, in der die Sprachstruktur in der Kindersprache aufgebaut wird, führt zu der Frage, in welcher Ordnung die Satzstruktur erworben wird. GUILFOYLE / NOONAN (1992) sowie MÜLLER (1993) argumentieren, dass zuerst die untergeordnete funktionale Kategorie der Flexion und die damit verbundene Projektion IP und dann die im Baumdiagramm oben gesetzte übergeordnete funktionale Kategorie des Komplementierers mit der dazugehörigen Projektion CP erworben werden. MÜLLER (1993) zeigt, dass in der deutschen und französischen Kindersprache zuerst korrekt flektierte Verben und korrekte Subjekte gebraucht werden, die als Anzeichen der IP fungieren, und erst in der nächsten Phase Nebensätze, also Evidenz für die CP, belegt werden können. TSIMPLI (2005:180) schlägt einen anderen Entwicklungsweg vor. Die Autorin nimmt an, dass interpretierbare Merkmale der funktionalen Kategorien vor den nicht-interpretierbaren erworben werden. ${ }^{11}$ Im Unterschied zur Strukturaufbauhypothese, nach der keine funktionale Kategorie am Anfang des Spracherwerbs Teil der kindlichen Grammatik ist, gehen CLAHSEN (1991a) und Clahsen / PENKE / PAROdI (1993/1994) davon aus, dass eine funktionale Kategorie gleich am Anfang des Spracherwerbs projiziert wird. Diese Kategorie wird als Finitheitsphrase bezeichnet, die weder mit der IP noch mit der CP in der Erwachsenensprache identisch ist.

\section{Funktionale Kategorien im Erwerb des Deutschen als Erstsprache}

Der Erwerb des Deutschen als Erstsprache ist ein Thema, das in der generativen Literatur seit mehreren Jahren berücksichtigt wird. ${ }^{12}$ Obwohl einige Studien einen gewissen Grad von individueller Variation in der Kindersprache aufzeigen, kann man ein allgemeines Entwicklungsmuster im Erwerb des

11 Die Unterscheidung in interpretierbare und nicht-interpretierbare Merkmale wurde im Minimalistischen Programm (CHOMSKY 1995) eingeführt. Während interpretierbare Merkmale relevant für die Interpretation auf der Ebene der Logischen Form (d.h. auf der Bedeutungsebene) sind, ist das bei den nicht-interpretierbaren Merkmalen nicht der Fall. Nicht-interpretierbare Merkmale werden im Verlauf der Derivation getilgt. Als Beispiel können hier die Person-, Numerus- und Genus-Merkmale von Nomina genannt werden, die als interpretierbar gelten, während die Person-, Numerus- und Genus-Merkmale von Verben als nicht-interpretierbar bezeichnet werden.

12 Vgl. Clahsen (1982, 1991a); Clahsen / Eisenbeiss / PenKe (1996); Clahsen / Kursawe / Penke (1996); Clahsen / PenKe (1992); Fritzenschaft / GAWlitzeKMaiwald / Tracy (1990); LasSer (2002); Meisel (1990, 1994, 1995); MülleR (1993); POEPPEL / WEXLER (1993); RothWEILER (1993); TRACY (1991, 2002). 
Aldona Sopata

Deutschen als Erstsprache beobachten. In der frühen Phase des Erwerbs des Deutschen als Erstsprache formulieren Kinder oft Äußerungen, die nicht-finite Verbalelemente anstelle von finiten Verben (sog. „Root-Infinitive“) beinhalten. ${ }^{13}$ In dieser Phase werden von den Kindern auch Äußerungen produziert, in denen finite Verben vorkommen. Bei diesen Verben handelt es sich meistens um Modalverben, Hilfsverben und Formen des Verbs sein. Die finiten Verben tauchen in der zielsprachigen Verb-Zweit-Stellung auf. Wegen der nicht zielsprachigen Infinitive wird diese Sprachentwicklungsstufe die Phase der optionalen Infinitive - „optional infinitive stage“ (WEXLER 1994) genannt. Charakteristisch für diese Phase ist auch das Auslassen von Subjekten, die in der deutschen Erwachsenensprache obligatorisch sind. ${ }^{14}$ Kinder benutzen in dieser Zeit keine Komplementierer, keine Nebensätze und keine W-Fragewörter. Eine detaillierte und überzeugende Hypothese zum Aufbau von funktionalen Kategorien im Erwerb des Deutschen als Erstsprache stellte MEISEL (1994) anhand von bilingualen Daten Deutsch/Französisch auf. Die ersten Mehrwortäußerungen, die meistens das Muster S+O oder S+Adjektiv aufweisen, werden dem Autor zufolge nicht durch morphosyntaktische Prinzipien geregelt. Die Entdeckung der lexikalischen Kategorie des Verbs durch das Kind ist der wichtigste Schritt hin zu den grammatischen Strukturen. Im Alter von ungefähr zwei Jahren tauchen in der Kindersprache die finiten Verbalformen auf. Die ersten Subjekt-Verb-Konstruktionen sind meistens Äußerungen wie beispielsweise:

Das ist [a] mann (Iv 2;0,2)

,das ist ein Mann“

(MEISEL 1994:95)

Die lexikalische Kategorie des Verbs wird im Rahmen der Hypothese als Kern der frühen grammatischen Strukturen angesehen. Die Mehrwortäußerungen in dieser Entwicklungsphase werden als Verbalphrasen mit den in der Spezifizierer-Position basisgenerierten Subjekten interpretiert. Ob die Verbalphrase zu diesem Zeitpunkt links- oder rechtsköpfig ist, kann nach Meisel noch nicht eindeutig entschieden werden. Die Identifizierung der lexikalischen Kategorie des Verbs ist für die frühe Phase des Grammatikerwerbs von höchstem Belang.

13 Vgl. LASSER (2002) für einen Überblick zu diesem Thema.

14 Abgesehen von Äußerungen, in denen das Expletivum es ausgelassen wird, und von dem sog. Topik-drop (das Auslassen von Subjekten in der Anfangsposition eines Satzes, das nur unter bestimmten Bedingungen in der Umgangssprache möglich ist). 
Funktionale Kategorien tauchen in der Kindergrammatik sukzessive auf, weil der Lerner erst entdecken muss, welche funktionale Kategorien und welche Merkmale dieser Kategorien Teil der von ihm zu erwerbenden Sprache sind. Ein wichtiges Indiz für den Erwerb einer funktionalen Kategorie ist der Erwerb der Finitheit. MEISEL (1994:98-100) zeigt, dass dieser Erwerb in zwei Phasen eingeteilt werden kann. In der ersten Phase, die ungefähr drei Monate dauert, kommen alle Formen vor, die die dritte Person Singular im Deutschen markieren (d.h. $O$ und $-t)$. Am Ende dieser Phase tauchen auch die Formen der ersten Person Singular bei Modalverben auf. Alle anderen Markierungen (d.h. erste Person Singular, zweite Person Singular, erste und dritte Person Plural: -0, -st, - $e$ und -en) kommen in der nächsten Entwicklungsstufe vor. Sie dauert auch ungefähr drei Monate. Nur einige unregelmäßige Formen und die Form der zweiten Person Plural werden später erworben. Wenn man bedenkt, dass die Kinder in dieser Zeit auch nicht-finite Verbalformen (Infinitive und Partizipien) sowie Formen des Imperativs benutzen, dann muss man bemerken, dass der Erwerb der Finitheit sehr schnell geschieht.

Tab. 1: Erwerb der Finitheit im Deutschen (nach MeISEL 1994:98)

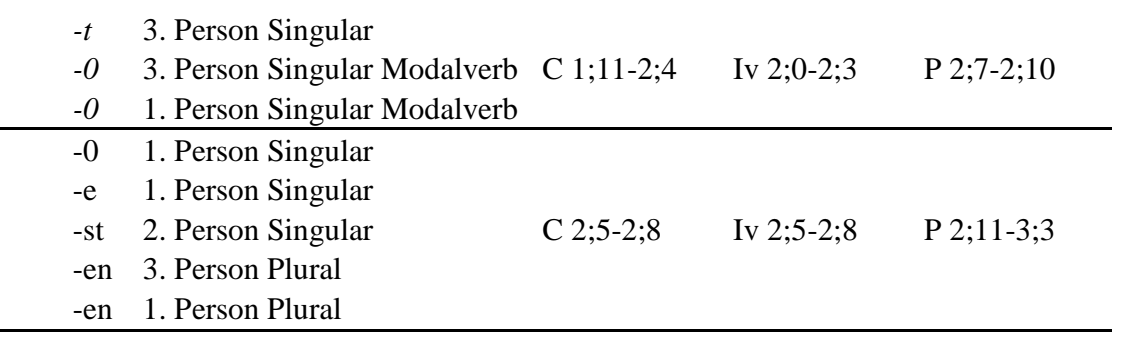

Um entscheiden zu können, ob die Finitheitsmarkierungen auch die Markierungen der Kongruenz sind, muss man die Distribution der Subjekte in der Kindersprache untersuchen. Meisel geht von der Annahme aus, dass man von dem Erwerb des syntaktischen Phänomens der Kongruenz sprechen kann, wenn das Verb mit dem Subjekt korrekt kongruiert und wenn die entsprechende Verbalform produktiv benutzt wird. Der Autor zeigt, dass die Verbalformen in der ersten Phase der Sprachentwicklung keine Kongruenzmarkierungen aufweisen und oft auch ohne Subjekt auftauchen. Sobald die finiten Verbalformen in der Kindersprache erscheinen, kongruieren sie mit den jetzt fast immer erscheinenden Subjekten, was als Indiz für ein grammatisches Phänomen gedeutet wird.

Die generative Sprachtheorie sagt voraus, dass Kinder mit dem Erwerb der Finitheit auch andere syntaktische Eigenschaften der gegebenen Sprache er- 
Aldona Sopata

werben sollten. Die wichtigste ist die Bewegung des Verbs zur Kopfposition der funktionalen Kategorie der Flexion (IP). Die Stellung der finiten Verbalelemente ist also ein starkes Indiz dafür, ob die die Verbalphrase determinierende funktionale Kategorie IP in die Struktur der Kindersprache eingebaut worden ist. Meisels erste wichtige Beobachtung in diesem Zusammenhang ist, dass die nicht-finiten Verbalformen immer in der Endstellung erscheinen. Sie werden also nie inkorrekt in die funktionale Kategorie nach oben bewegt und erscheinen daher nie in der Zweitstellung. Im Gegensatz dazu kommen die Verben mit Finitheitsmarkierungen meistens in der korrekten V2-Stellung nach den Subjekten oder anderen topikalisierten Konstituenten im Alter von 2;4-2;5 vor, was die Evidenz für den Aufbau der funktionalen Kategorie der Flexion ist.

(6) kaputt is der (Iv 2;04,09)

ein grüner is das (C 2;04,22)

da fährt die Caroline (C 2;03,11)

(MEISEL 1994:107f.)

Ein anderes wichtiges Phänomen, das bei der Untersuchung der funktionalen Kategorie IP berücksichtigt werden muss, ist die Stellung der Verben in Bezug auf die Negation. Im Deutschen stehen die finiten Verben (in Hauptsätzen) vor und die nicht-finiten Verbalelemente nach der Negation nicht. Im Alter von 2;4 Jahren ist diese Erscheinung in den von Meisel untersuchten Daten zu sehen:

(7) de-de-des geht nicht (Iv 2;04,09)

Ivar darf nich tee (Iv 2;04,23)

passt nich auch (Iv 2;05,07)

(MEISEL 1994:108)

In dieser Entwicklungsphase beginnen in der Kindersprache auch die Modalverben zu erscheinen, was auch von Bedeutung ist, da die Modalverben in einer funktionalen Kategorie (IP oder T) generiert werden ${ }^{15}$ und die nicht-finiten Verbalelemente in der Verbalphrase bleiben. Das Erscheinen der Modalverben ist also auch ein Indiz dafür, dass die IP implementiert worden ist. Man muss unterstreichen, dass alle besprochenen Phänomene, die als Folge des Einbaus der funktionalen Kategorie IP zu interpretieren sind, in derselben Phase der

15 Die Annahme wird dadurch unterstützt, dass es keine „optionalen“ Infinitive bei Modalverben in der Kindersprache gibt. Sie sind entweder finit oder tauchen gar nicht auf. 
Sprachentwicklung der Kinder im Alter von 2;3-2;4 (C), 2;4-2;5 (Iv) oder 2;11-3;0 (P) vorkommen. MeISEL (1994:109) unterstreicht, dass diese Phase auch jeweils die Zeit ist, in der die Kinder andere Personmarkierungen als die dritte Person Singular zu benutzen beginnen. Die Kategorie Tempus kommt erst in der nächsten Phase der Sprachentwicklung. Der Erwerb der Finitheit ist also nach Meisel in erster Linie mit der Subjekt-Verb-Kongruenz verbunden, und diese ist der Auslöser für den Aufbau der funktionalen Kategorie, die die Verbalphrase subkategorisiert. ${ }^{16}$ Auch andere Untersuchungen zum Aufbau der funktionalen Kategorie IP im Deutschen zeigen, dass es ein schneller und einheitlicher Prozess ist. Im Allgemeinen wird festgestellt, dass weniger als $10 \%$ der finiten Verben in der End-Stellung erscheinen und nur 1\% - 2\% der nichtfiniten Verben in der Zweit-Stellung vorkommen (CLAHSEN / EISENBEISS / PENKE 1996). Der Gebrauch der finiten Verben in der Endstellung wird als zulässige individuelle Variation verstanden (TRACY 2002:678f.). Im Deutschen kann man in dieser Phase auch beobachten, dass das Auslassen der Subjekte stark abnimmt und dann verschwindet (HAMANN 1996:199-201).

Der nächste Schritt im Erwerb des Deutschen als Erstsprache ist der Aufbau der weiteren funktionalen Kategorie, d.h. der Komplemetiererphrase (CP). Es wird angenommen, dass die vollständig spezifizierte Komplementiererphrase erst dann in der Kindersprache identifiziert werden kann, wenn das Kind topikalisierte Objekte und Fragen mit W-Fragewörtern gebraucht. Ein weiteres Indiz für die aufgebaute CP sind lexikalische Komplementierer und Nebensätze, die vom Kind produziert werden. Diese Erwerbsphase beginnt entweder gleichzeitig oder kurz nach der Phase des Aufbaus der Flexionsphrase. ${ }^{17}$ Die lexikalischen Auslöser für die Implementierung der CP sind Komplementierer. Nachdem sie vom Kind identifiziert worden sind, verläuft der Erwerb der Nebensätze fast fehlerlos (MEISEL 1986; ROTHWEILER 1993). Nur einige Kinder durchlaufen eine kurze Übergangsphase, in der sie Nebensätze mit finiten Verben in der Endstellung und fehlenden Komplementierern benutzen (FRITZENSChafT / GaWlitZeK-MaiwaLd / TRACY 1990; MüLLER 1993). Mit dem

16 Die Beobachtung unterstützt die sog. Split-INFL-Hypothese von PoLLOCK (1989), die die funktionale Kategorie IP in die untergeordnete Kategorie der Kongruenz (AGR) und die übergeordnete Kategorie des Tempus (T) spaltet.

17 Vgl. Clahsen / Kursawe / PenKe (1996); FritZENSChaft / GAWLITZEK-Maiwald / TRACY (1990); ROTHWEILER (1993). 
Aldona Sopata

Aufbau der Komplementiererphrase ist die Konstruktion der Sprachstruktur im Verbalbereich im Deutschen abgeschlossen. ${ }^{18}$

\section{Funktionale Kategorien im Erwerb des Polnischen als Erstsprache}

Der Erwerb des Polnischen als Erstsprache ist ein Forschungsgebiet, in dem in den letzten Jahrzehnten einige Arbeiten erschienen sind. ${ }^{19}$ Es gibt jedoch kaum Untersuchungen zum Erwerb des Polnischen als Erstsprache, die die Einsichten der generativen Theorie in den Strukturaufbau der menschlichen Sprache berücksichtigen würden. ${ }^{20}$ Die erste Phase des Strukturaufbaus im Erwerb des Polnischen als Erstsprache sieht anders aus als im Erwerb des Deutschen. BAR SHALOM / SNYDER (1997:25f.) stellen fest, dass in der frühen Kindersprache im Polnischen kaum Infinitive anstelle der finiten Verbalformen (sog. ,RootInfinitives“) vorkommen. Anhand einer Untersuchung der Daten von Kindern aus der CHILDES-Datenbank (MAC WHINNEY / SNOW 1985) zeigen sie, dass von 4.519 Kinderäußerungen nur 9 Verben als optionale Infinitive analysiert werden können. Das steht im Gegensatz dazu, was nicht nur im Deutschen, sondern auch beispielsweise im Russischen oder Italienischen geschieht. Diese Beobachtung wird dadurch erklärt, dass sich das Polnische hier vom Deutschen und vom Russischen in sehr wesentlichen Punkten unterscheidet. Die Phase der optionalen Infinitive tritt in den Sprachen auf, in denen die Verbalmorphologie nicht reich ist oder in denen Null-Subjekte nicht zugelassen sind (RHEE / WEXLER 1995). Während Deutsch und Russisch zumindest das zweite Kriterium erfüllen, tritt keines der Phänomene im Polnischen auf. Problematischer sieht der Unterschied zum Italienischen aus, da beide Sprachen ein reiches Verbalparadigma haben und beide Null-Subjekt-Sprachen sind. HOEKSTRA / HyAMS (1998) zeigen jedoch, dass der Anteil der Root-Infinitive in der frühen Phase des Erstspracherwerbs bei Null-Subjekt-Sprachen zwischen $3 \%$ und $16 \%$ liegen kann und dass diese Entwicklungsstufe relativ kurz ist. TRYZNA (2007:637) zeigt anhand einer Analyse von anderen Kindern aus der CHILDES-Datenbank, dass der Anteil der Root-Infinitive bei den von ihr analysierten Kindern zwischen 9\% und 14\% liegt. Dieser Wert ist mit den Da-

18 Auf den Strukturaufbau im Nominalbereich wird in diesem Artikel nicht eingegangen.

19 Dazu gehören u. a. ŁUCZYŃSKI (2004); PRZETACZNIK-GIEROWSKA (1994); SHUGAR / SMOCZYŃSKA (1980); SMOCZYŃSKI (1955); SZUMAN (1968); ŚWIĘCICKA (1993).

20 Auf die wenigen vorhandenen Studien zum Erwerb des Polnischen als Erstsprache im generativen Paradigma wird im Artikel weiter eingegangen. 
ten aus dem Italienischen vergleichbar. Die vorliegende Studie stützt sich auch auf die longitudinalen Daten aus der CHILDES-Datenbank (MACWHINNEY / SNOw 1985). Es werden die Daten von zwei Kindern, Basia (Alter 1;7-2;0) und Kasia (Alter 1;4-1;9), analysiert. Jede Datei beinhaltet Aufnahmen, die während eines Monats gemacht worden sind. Die ersten ein Verb enthaltenden Mehrwortäußerungen im Polnischen sind beispielsweise folgende Wortketten:

$$
\begin{aligned}
& \text { dać kul kul (Kas1;4) } \\
& \text { geben kul kul=Ball } \\
& \text {,einen Ball geben` } \\
& \text { dzidzi pisie (Kas 1;4) } \\
& \text { dzidzi=Kind schreibt } \\
& \text {,das Kind schreibt‘ }
\end{aligned}
$$

$$
\text { boi kicie (Kas 1;4) }
$$

Angst haben - 3. Person Singular kicie=Katze ,Ich habe Angst vor der Katze“

Das Beispiel (8a) zeigt den Gebrauch eines „Root-Infinitives“ im Polnischen, weil eine non-finite Verbalform anstelle einer finiten auftritt. Im Beispiel (8b) taucht ein finites Verb auf. Es handelt sich dabei eindeutig um die dritte Person Singular, was auch durch das vorstehende Nomen bestätigt wird. Im Beispiel (8c) haben wir es mit einem Null-Subjekt zu tun, das auch in der polnischen Erwachsenensprache häufig vorkommt. Das Verb ist finit, es ist jedoch 3. Person Singular, und von der Situation her ist es ziemlich eindeutig, dass das Kind sich selbst meint. In der Erwachsenensprache würde das Verb in der 1. Person Singular benutzt. Da kein Nomen an der Stelle des Subjekts vorkommt, haben wir es natürlich nicht mit einem Kongruenzfehler zu tun. Die Situation kann so interpretiert werden, dass in dieser Phase der Sprachentwicklung vom Kind noch keine funktionalen Kategorien aufgebaut worden sind. ${ }^{21}$ Es sind Verbalphrasen, in denen das Verb als Infinitiv oder als 3. Person Singular realisiert wird. Die 3. Person Singular wird als unmarkierter Wert (,default ${ }^{\dagger}$-Wert) verstanden (vgl. MeISEl 1994:115; PlatZaCK / Holmberg 1989:58). ${ }^{22}$ Die

21 Im Beispiel (8c) haben wir es zusätzlich mit der Auslassung des Reflexivpronomens się - sich zu tun, was in dieser Entwicklungsstufe häufig vorkommt. Auf die Interpretation des Phänomens kann im Rahmen dieses Artikels nicht eingegangen werden.

In den Grammatiken unterschiedlicher Sprachen wird die 3. Person Singular bei expletiven oder unpersönlichen Subjekten gebraucht. Zum Beispiel im Deutschen: ihrer wurde gedacht vs. *ihrer wurden gedacht (vgl. MeISEL 1994:15). 
Verbalphrasen in dieser Entwicklungsstufe zeigen jedoch schon eine zielsprachige Wortfolge, d.h. eine VO-Folge (s. Beispiel 8a), was darauf schließen lässt, dass die Verbalphrase in der polnischen Kindersprache von Anfang an linksköpfig ist. ${ }^{23}$

Im Polnischen wird die Finitheit ähnlich wie im Deutschen durch Personalendungen ausgedrückt, die folgendermaßen lauten:
1. Person Singular
-ę/-m
1. Person Plural -my
2. Person Singular
$-\mathrm{sZ}$
2. Person Plural -cie
3. Person Singular
$-0$
3. Person Plural - ą

Die vielfältigen anderen Unterschiede im Verbalparadigma können auf Stammsuffixe reduziert werden (vgl. NAGÓRKO 1998:115-119). ${ }^{24}$ In der polnischen Kindersprache tritt die Form der 3. Person Singular sehr früh auf, was in den Beispielen (8b), (8c) zu sehen ist. Auch Markierungen für andere Personen werden im Polnischen von den Kindern relativ früh erworben. Bei dem ersten Kind, Basia, dessen sprachliche Äußerungen hier analysiert werden, kann man feststellen, dass es im Alter von 1;7, 1;8 und 1;9 die 3. Person Singular produktiv benutzt ${ }^{25}$ :

(a)

\author{
dzidzi pii \\ (Bas $1 ; 7)$ \\ dzidzi=Kind schlafen (3. Singular) \\ ,das Kind schläft‘
}

23 Vgl. den Strukturbaum des Polnischen unter Punkt 3.

24 Je nach der morphologischen Struktur des Verbs können drei Arten der Konjugation unterschieden werden:

1. Person Singular (-ę,-ę,-m), 2. Person Singular (-esz, -isz, -sz), 3. Person Singular (-e, -i, -0), 1. Person Plural

(-emy, -imy, -my), 2. Person Plural (-ecie, -icie, -cie) und 3. Person Plural (-ą, -ą, a) (nach NAGÓRKO 1998:118f.).

25 Im vorliegenden Artikel wird davon ausgegangen, dass eine Form produktiv gebraucht wird, wenn sie mit mehr als einem Verb vorkommt und in mehreren aufeinanderfolgenden Aufnahmen auftaucht. 
Funktionale Kategorien im Erwerb des Deutschen und des Polnischen
(b)
lado ga
(Bas 1;8)
lado=Radio spielen (3. Singular)
,das Radio spielt'
(c) tu jeś тии
hier ist (3. Singular) muu=Kuh
,hier ist eine Kuh'

Auf dieser Entwicklungsstufe (1;7-1;9) treten bei Basia auch vereinzelt Markierungen für andere Personen (2. Singular und 3. Plural) auf. Sie können aber nicht als produktiver Gebrauch betrachtet werden, da sie nur mit einzelnen Verben vorkommen. Im Alter von 1;10 beginnt Basia die Markierung für die 2. Person Singular produktiv zu benutzen, und im Alter von 1;11 tauchen alle anderen Personalendungen außer der 2. Person Plural in Basias Verbalparadigma auf:

$\begin{array}{lll}\text { (10) (a) } \quad \begin{array}{l}\text { idziś misiu } \\ \text { siehst Teddy } \\ \text {,siehst du, Teddy }\end{array} & \\ & \text { potem leciom } \\ \text { (b) } & \text { (Bann fliegen (3. Plural) } \\ & \text {,dann fliegen sie ‘ } \\ & \text { ziupe bedziemy jeść? (Bas 1;11) } \\ & \text { Suppe-Akk werden (1. Plural) essen } \\ & \text {,werden wir die Suppe essen? }\end{array}$

Bei dem zweiten untersuchten Kind, Kasia, verläuft der Erwerb der Personmarkierungen noch schneller. Schon im Alter von 1;04 gebraucht Kasia produktiv die Personalendung für die 3. Person Singular (Beispiel 11a). Dabei muss man jedoch anmerken, dass die Formen der 3. Person Singular im Polnischen auch als Verbalstämme interpretiert werden können. In dieser Phase treten bei Kasia auch vereinzelte Beispiele auf, die als Markierungen der 1 . Person Singular interpretiert werden könnten. Es handelt sich aber dabei um Fälle, in denen aus phonetischen Gründen die Form der 1. Person von der 3. Person nicht zu unterscheiden ist. ${ }^{26}$ Im Alter von 1;7 beginnt Kasia die 1. und die 2. Person Singular (Beispiel 11b) zu benutzen. Darauf folgen im Alter von 1;8 die 1. und 3. Person Plural (Beispiel 11c).

26 Zum Beispiel kann pisie (Kas 1;6), ich schreibe = er/sie/es schreibt ‘ sowohl für die 1. als auch für die 3. Person Singular fungieren, da die eigentliche Personalendung für die 1. Person (-ę) auch von Erwachsenen als -e ausgesprochen wird. 
Aldona Sopata

(11) (a)

$$
\text { patrzi }
$$

(Kas $1 ; 4)$

sieht (3. Singular)

er/sie/es sieht ${ }^{\star}=$ Kasia will aus dem Fenster sehen (situationsabhängig)

(b) idzis?

(Kas $1 ; 7)$

siehst?

, siehst du?'

(c) zialaś źlobimy taka jaje, chwapcika

(Kas $1 ; 8)$

gleich machen (3. Plural) Futur eine Puppe-Akk, ein Junge-Akk

,gleich machen wir eine Puppe - einen Jungen“

Zusammenfassend kann der Erwerb der Finitheit wie folgt dargestellt werden:

Tab. 2: Erwerb der Finitheit im Polnischen

\begin{tabular}{llll}
\hline-0 & 3. Person Singular & Basia $1 ; 7-1 ; 9$ & Kasia $1 ; 4-1 ; 6$ \\
\hline -sz & 2. Person Singular & Basia $1 ; 10$ & Kasia $1 ; 7$ \\
-ę/-m & 1. Person Singular & Basia $1 ; 11$ & Kasia $1 ; 7$ \\
-my & 1. Person Plural & Basia $1 ; 11$ & Kasia $1 ; 8$ \\
-ą & 3. Person Plural & Basia $1 ; 11$ & Kasia $1 ; 8$ \\
\hline
\end{tabular}

Polnisch ist eine Null-Subjekt-Sprache. Die Null-Subjekte treten auch in der Erwachsenensprache sehr häufig auf, daher kann das Vorkommen der Subjekte in kindlichen Äußerungen im Unterschied zum Deutschen keine Evidenz für den Erwerb des syntaktischen Phänomens der Kongruenz sein. In den Fällen, in denen die Subjekte realisiert werden, kongruieren sie jedoch korrekt mit dem Verb.

Polnisch ist keine V2-Sprache, und die Stellung der Verben im Satz ist relativ frei. Die Stellung der finiten und nicht-finiten Verbalelemente im Satz ist also im Polnischen im Unterschied zum Deutschen keine Evidenz für den Aufbau der funktionalen Kategorie. Ein Phänomen, das jedoch einen Einblick in die mentale Grammatik des Kindes gewähren und auch zeigen kann, ob eine die Verbalphrase dominierende funktionale Kategorie in die kindliche Grammatik wirklich implementiert wurde, ist die Stellung der Verben in Bezug auf die Negation. Es wird angenommen, dass die Negation im Polnischen ein funktionaler Kopf ist. Die Negationsphrase (NegP) steht zwischen der AgrP und TP 
(vgl. PILARSKI 2002:127). Das erklärt die Tatsache, dass die Negation in der polnischen Erwachsenensprache immer eine Position vor dem Verb besetzt. In der Kindersprache wird die Negation zuerst außerhalb der Verbalphrase eingesetzt:

nie pama boi, nie

(Basia 1;9)

nicht den Mann-Akk fürchten (3. Singular), nicht ,ich fürchte nicht den Mann`

(b)

gyzie nie
beißt nicht
, sie beißt nicht"

Im Beispiel (12a) haben wir es mit der Wortfolge Neg-Objekt-Verb zu tun, die keine kanonische Wortfolge des Polnischen ist und hier als ungrammatisch bezeichnet werden muss. ${ }^{27}$ Im Beispiel (12b) besetzt die Negation die Position hinter dem Verb, was in der polnischen Erwachsenensprache nie vorkommt. Bemerkenswert ist die Tatsache, dass diese von der Erwachsenennorm abweichenden Formen verschwinden, sobald von den Kindern die Finitheit erworben ist. Beim ersten untersuchten Kind (Basia) wird die Negation im Alter von 1;10 korrekt eingesetzt, d. h. in dem Alter, in dem das Kind die Markierung für die 3. und 2. Person Singular produktiv benutzt. Das zweite Kind (Kasia) setzt die Negation in die Position vor dem Verb im Alter von 1;9 ein, d.h. unmittelbar nachdem das Kind die Finitheit (d.h. die Markierungen für die 1., 2., 3. Person Singular und die 1. und 3. Person Plural) erworben hat (Tabelle 2). Der korrekte Einsatz der Negation ist daher zweifellos eine Folge des Einbaus der funktionalen Kategorien (IP und $\mathrm{NegP})^{28}$ in die kindliche Grammatik.

\section{Schlussfolgerungen}

Der Erwerb des Deutschen und des Polnischen als Erstsprache folgt ähnlichen Entwicklungsprinzipien. In beiden Sprachen ist der Erwerb der Finitheit der Auslöser für den Aufbau der funktionalen Kategorie, die die Verbalphrase

27 Nur unter bestimmten Bedingungen könnte der Satz mit dem negierten topikalisierten Objekt in der polnischen Erwachsenensprache vorkommen - Nicht den Mann fürchte ich, sondern einen anderen.

28 In diesem Artikel kann weder auf den Erwerb der funktionalen Kategorie Tempus eingegangen werden, der im Polnischen gleichzeitig mit dem Erwerb der Kongruenz geschieht, noch auf die weitere Sprachentwicklung, in der die CP aufgebaut wird. 
Aldona Sopata

unterordnet. In beiden Sprachen geschieht der Erwerb ziemlich schnell. Im Polnischen setzt er früher an, was jedoch angesichts der Tatsache, dass Polnisch über eine sehr reiche Verbalmorphologie verfügt, zu erwarten ist. Die Dauer der Entwicklungsstufe ist im Deutschen und im Polnischen sehr ähnlich (5-6 Monate). In beiden Sprachen kann man auch den Erwerb der Finitheit in zwei Phasen einteilen. In der ersten Phase (3 Monate) wird nur die Markierung für die 3. Person Singular gebraucht, in der zweiten kommen dann alle anderen Personenmarkierungen (außer der 2. Person Plural) in der Kindersprache vor. Sowohl im Deutschen als auch im Polnischen handelt es sich beim Erwerb der Finitheit um ein komplexes syntaktisches Phänomen, das Folgen für den Aufbau des gesamten Verbalbereichs hat. Im Deutschen ist die Folge der Erwerb der V2-Stellung, die entsprechende Distribution der finiten und der nicht-finiten Verbalelemente sowie die Stellung des Verbs in Bezug auf die Negation. Im Polnischen ist der Aufbau der funktionalen Kategorien dadurch zu erkennen, dass die Negation in der Kindersprache die entsprechende Position vor dem Verb einnimmt. Zusammenfassend kann man also sagen, dass die funktionalen Kategorien im Erstspracherwerb des Deutschen und Polnischen ähnlich aufgebaut werden, obwohl sich die Entwicklung der mentalen Grammatik des Kindes in den sprachlichen Phänomenen der Einzelsprachen auf unterschiedliche Weise widerspiegelt.

Betrachtet man die oben dargestellte Sprachentwicklung im Deutschen und im Polnischen näher, bemerkt man, dass der Verlauf des Erstspracherwerbs am besten durch den Strukturaufbau zu erklären ist. Die zeitliche Korrelation des Erwerbs verschiedener Phänomene in unterschiedlichen Sprachen kann ja kein Zufall sein. Sie ist eher ein Verhältnis von Ursache und Wirkung. Der Aufbau einer funktionalen Kategorie hat den Erwerb von mehreren syntaktischen Phänomenen zur Folge. Dieser Zusammenhang wird verständlich, wenn man die Hypothese des Strukturaufbaus (GUILFOYLE / NOONAN 1992) als Grundlage der Erklärung des Erstspracherwerbs annimmt. In der Reifungshypothese (BORER / WEXLER 1987) ist die Korrelation weniger plausibel, da die Hypothese die Sprachentwicklung von der neurophysiologischen Reifung abhängig macht. Die im Artikel dargestellten Daten können kaum durch die Hypothese der vollen Kompetenz (POEPPEL / WEXLER 1993; BORER / ROHRBACHER 2002) erklärt werden, weil ihre Vertreter annehmen, dass dem Kind von Beginn an alle funktionalen Kategorien zur Verfügung stehen. Die sprachliche Entwicklung der Kinder, die Deutsch und Polnisch als ihre Muttersprache erwerben, zeigt das Gegenteil. Auch wenn die Kinder finite Verben sehr früh benutzen, dauert es eine Zeit lang, bis sie alle mit der entsprechenden funktionalen Kategorie zusammenhängenden Phänomene erworben haben. Man sieht in beiden 
Sprachen, dass die Kinder erst dann über die vollständig spezifizierte funktionale Kategorie verfügen, wenn sie mehrere Personmarkierungen erworben haben.

$\begin{array}{ll}\text { Abkürzungen } \\ \text { AGR } & \text { Kongruenz } \\ \text { C } & \text { Kopfposition der Komplementiererphrase } \\ \text { CP } & \text { Phrase des Komplementierers } \\ {[+F]} & \text { Finitheitsmerkmal } \\ \text { I } & \text { Kopfposition der Flexionphrase } \\ \text { IP } & \text { Phrase der Flexion } \\ \text { N } & \text { Nomen } \\ \text { Neg } & \text { Negation } \\ \text { NegP } & \text { Phrase der Negation } \\ \text { O } & \text { Objekt } \\ \text { S } & \text { Subjekt } \\ \text { T } & \text { Tempus } \\ \text { UG } & \text { Universalgrammatik } \\ \text { V } & \text { Verb } \\ \text { VP } & \text { Verbalphrase } \\ \text { V2 } & \text { Verbzweitstellung }\end{array}$

\section{Literatur}

Bar Shalom, Eva / SNyder, William (1997): Root infinitives in Child Russian. A comparison with Italian and Polish. In: SHILlCock, Richard / Sorace, ANTONElla / Heycock, CARoline (eds.): Language Acquisition: Knowledge Representation and Processing. Proceedings of GALA '97. Edinburgh, 22-27.

Borer, Hagit / Rohrbacher, Bernhard (2002): Minding the absent. Arguments for the Full Competence Hypothesis. In: Language Acquisition 10 (2):123-175.

Borer, Hagit / WeXler, KenNeth (1987): The maturation of syntax. In: RoEPER, Thomas / Williams, Edwin (eds.): Parameter Setting. Dordrecht, 123-172.

CAZDEn, Courtney B. (1972): Child Language and Education. New York.

CHOMSкy, NoAm (1981): Lectures on Government and Binding. Dordrecht.

- (1986): Knowledge of Language. Its Nature, Origin, and Use. New York. 
Aldona Sopata

- (1989): Some Notes on Economy of Derivation and Representation. In: LAKA, ITZIAR / Mahajan, Anoop (eds.): Functional Heads and Clause Structure. MIT Working Papers in Linguistics 10:43-74.

- (1995): The Minimalist Program. Cambridge (Mass.).

Clahsen, Harald (1982): Spracherwerb in der Kindheit. Eine Untersuchung zur Entwicklung der Syntax bei Kleinkindern. Tübingen.

- (1991): Constraints on parameter setting. A grammatical analysis of some acquisition stages in German child language. In: Language Acquisition 1:361-391.

- (1991a): Child Language and Developmental Dysphasia: Linguistic Studies of the Acquisition of German. Amsterdam.

Clahsen, Harald / Eisenbeiss, Sonja / Penke, Martina (1996): Lexical learning in early syntactic development. In: ClAHSEN, HARALD (ed.): Generative Perspectives on Language Acquisition. Empirical Findings, Theoretical Considerations and Crosslinguistic Comparisons. Amsterdam, 129-159.

Clahsen, Harald / Kursawe, Claudia / Penke, Martina (1996): Introducing CP: wh-questions and subordinate clauses in German child language. In: KosTER, Charlotte / Wijnen, Frank (eds.): Proceedings of the Assembly on Language Acquisition [GALA 1995]. Groningen, 5-22.

Clahsen, Harald / PenKe, Martina (1992): The acquisition of agreement morphology and its syntactic consequences. New evidence on German child language from the Simone-corpus. In: MeISEL, JÜrgen M. (ed.): The Acquisition of Verb Placement. Functional Categories and V2 Phenomena in Language Acquisition. Dordrecht, 181-223.

Clahsen, Harald / Penke, Martina / Parodi, Teresa (1993/1994): Functional categories in early child German. In: Language Acquisition 3:395-429.

FELIX, SASCHA (1984): Maturational aspects of Universal Grammar. In: DAVIS, AlAN / CRIPPER, Clive / HowATT, ANTHONY (eds.): Interlanguage. Edinburgh, 133-161.

- (1992): Language acquisition as a maturational process. In: WEISSENBORN, JÜRGEN / GOODLuCK, Helen / RoEPeR, THOMAs (eds.): Theoretical Issues in Language Acquisition: Continuity and Change in Development. Hillsdale (NJ), 25-53.

Fletcher, PAul / MACWhinney, Brian (eds.) (1995): The Handbook of Child Language. Oxford.

Fritzenschaft, Agnes / Gawlitzek-Maiwald, Ira / Tracy, Rosemarie (1990): Wege zur komlexen Syntax. In: Zeitschrift für Sprachwissenschaft 9:52-134.

GleitMAn, LILA R. / WANNER, ERIC (1982): Language acquisition. The state of the state of the art. In: WANNER, ERIC / GLEITMAN, LILA R. (eds.): Language Acquisition: The State of the Art. Cambridge, 3-48.

GOOdLuCK, HeLEN (1986): Language acquisition and linguistic theory. In: FLETCHER, Paul / Garman, Michael (Hrsg.): Language Acquisition. Cambridge, 49-68.

GrewENdORF, GÜNTHER (1988): Aspekte der deutschen Syntax. Eine Rektions-Bindungs-Analyse. Tübingen. 
Funktionale Kategorien im Erwerb des Deutschen und des Polnischen

- (1992): Parametrisierung der Syntax. In: HofFMAnN, LudGER (ed.): Deutsche Syntax. Ansichten und Aussichten. Berlin, 11-73.

- (2002): Minimalistische Syntax. Tübingen.

Guilfoyle, Eithne / NoOnan, Maire (1992): Functional Categories and Language Acquisition. In: Canadian Journal of Linguistics 37:241-272.

Haegeman, Liliane (1991): Introduction to Government and Binding Theory. Oxford.

Hamann, CoRnelia (1996): Null Arguments in German Child Language. In: Language Acquisition 5:155-208.

Hoekstra, Teun / Hyams, Nina (1998): Aspects of Root Infinitives. In: Lingua 106:81-112.

HyAms, NinA (1986): Language and the Theory of Parameters. Dordrecht.

- (1987): The theory of parameters and syntactic development. In: ROEPER, THOMAS / Williams, Edwin (eds.): Parameter Setting. Dordrecht, 340-371.

LASSER, INGEBORG (2002): The roots of root infinitives. Remarks on infinitival main clauses in adult and child language. In: Linguistics 40:767-796.

ŁUCZYŃSKI, EDWARD (2004): Kategoria przypadka w ontogenezie języka polskiego czyli $O$ wchodzeniu dziecka w rzeczywistość gramatycznq. [Die Kasuskategorie in der Ontogenese des Polnischen also Über den Eingang des Kindes in die grammatische Wirklichkeit]. Gdańsk.

MAC Whinney, BRIAn / SNOw, CATHERINE (1985): The child language exchange system. In: Journal of Child Language 12:271-296.

MeCNER, PAWEŁ (1995): Vorstudien zur generativen Morphosyntax des Polnischen. Das X-bar Schema des Verbkomplexes (Finitparadigma). In: Linguistica Silesiana 16:79-86.

- (1997): Skizzen zur generativen Morphosyntax des Polnischen im Vergleich mit dem Deutschen. In: Colloquia Germanica Stetinensia 7:5-26.

- (2005): Elementy gramatyki umystu. Od struktur sktadniowych do Minimalizmu. [Grundzüge der mentalen Grammatik. Von den syntaktischen Strukturen zum Minimalismus]. Kraków.

MEISEL, JÜRGEN M. (1986): Word order and case marking in early child language. Evidence from the simultaneous acquisition of two first languages: French and German. In: Linguistics 24:123-183.

- (1990): INFL-ection: Subjects and Subject-Verb Agreement in Early Child Language. In: MeISEL, JÜRGEN M. (ed.): Two First Languages. Early Grammatical Development in Bilingual Children. Dordrecht, 237-298.

- (1994): Getting FAT: The role of finiteness, agreement and tense in early grammars. In: MeISEl, JÜRgen M. (ed.): Bilingual First Language Acquisition. French and German Grammatical Development. Amsterdam, 89-129.

- (1995): Parameters in Acquisition. In: FleTCHER / MACWHINNEY, 123-156. 
Aldona Sopata

Müller, Natascha (1993): Komplexe Sätze. Der Erwerb von COMP und von Wortstellungsmustern bei bilingualen Kindern (Französisch/Deutsch). Tübingen.

NaGóRKo, Alicja (1998): Zarys gramatyki polskiej. [Abriss der polnischen Grammatik]. Warszawa.

Otsu, Yukio (1981): Universal Grammar and Syntactic Development in Children. Cambridge (Mass.).

Pilarski, AnNa (2002): Die Operation Merge im Verbalkomplex des Polnischen und des Deutschen. Frankfurt (M.).

Pinker, STEven (1984): Language Learnability and Language Development. Cambridge. - (1994): How could a child use verb syntax to learn verb semantics? In: Lingua 92:377-410.

Platon (1991): Menon, Kratylos, Euthydemos, Hippias Maior. Griechisch und Deutsch. In: Sämtliche Werke. Bd. III. Frankfurt (M.).

Platzack, Christer / Holmberg, ANDERs (1989): The role of AGR and finiteness in Germanic VO Languages. In: Scandinavian Working Papers in Linguistics 43:51-76.

Poeprel, David / WeXler, Kenneth (1993): The Full Competency Hypothesis of clause structure in early German. In: Language 69:1-33.

POLLOCK, JEAN Yves (1989): Verb movement, universal grammar and the structure of IP. In: Linguistic Inquiry 20:365-424.

PRZETACZNIK-GIEROwSKa, MARIa (1994): Od stowa do dyskursu: studia nad mowa dziecka. [Vom Wort zum Diskurs: Studien zur Kindersprache]. Warszawa.

RADFORD, ANDREW (1990): Syntax Theory and the Acquisition of Syntax. Oxford.

- (1995) Phrase structure and functional categories. In: Fletcher / MACWhinNey, 483-507.

- (1997): Syntactic Theory and the Structure of English. A Minimalist Approach. Cambridge (Mass.).

Rhee, Jaemin / WeXler, Kenneth (1995): Optional Infinitives in Hebrew. In: MIT Working Papers in Linguistics 26:383-402.

Rothweiler, Monika (1993): Der Erwerb von Nebensätzen im Deutschen. Tübingen.

Shugar, Grace / SMOCZyŃsKa MAgdalena (1980): Badania nad rozwojem języka dziecka. Wybór prac. [Untersuchungen zur Entwicklung der Kindersprache. Auswahl von Arbeiten]. Warszawa.

SMOCZYŃSKI, PAwEe (1955): Przyswajanie przez dziecko podstaw systemu językowego. [Der kindliche Erwerb der Grundlagen des Sprachsystems]. Wrocław.

SzUMAn, Stefan (ed.) (1968): O rozwoju języka i myślenia dziecka. [Über die Entwicklung der Sprache und des Denkens des Kindes]. Warszawa.

ŚwIĘCICKA, MAŁGORZATA (1993): Charakterystyka sktadniowa wypowiedzi dzieci $w$ wieku przedszkolnym. [Syntaktische Charakteristik der Äußerungen von Kindern im Kindergartenalter]. Bydgoszcz. 
Funktionale Kategorien im Erwerb des Deutschen und des Polnischen

Tracy, Rosemarie (1991): Sprachliche Strukturentwicklung. Linguistische und kognitions-psychologische Aspekte einer Theorie des Erstspracherwerbs. Tübingen.

- (2002): Growing (clausal) roots: all children start out (and remain) multilingual. In: Linguistics 40:653-686.

TRYZna, MARTA (2007): Root infinitives in early child Polish: the syntactic and interpretative properties. In: Proceedings of the 31st Annual Boston University Conference of Language Development. Somerville, 632-643.

Tsimpli, IANTHI-Maria (2005): Peripheral positions in early Greek. In: Stavrou, Melita / Terzi, Arhonto (eds.): Advances in Greek Generative Syntax: Synchrony, Diachrony and Acquisition. Amsterdam/Philadelphia, 176-216.

WEXLER, KENNETH (1994): Optional infinitives, head movement and economy of derivations. In: LightFOOT, DAVID / HoRnsteIn, Norbert (eds.): Verb Movement. Cambridge, 305-350. 